\title{
Oscillatory Regime in Excitatory Media with Global Coupling: Application to Cardiac Dynamics
}

\author{
E Alvarez-Lacalle ${ }^{1}$, JF Rodriguez ${ }^{2}$, B Echebarria ${ }^{1}$ \\ ${ }^{1}$ Universitat Politecnica de Catalunya, Departament de Fisica Aplicada, Barcelona, Spain \\ ${ }^{2}$ Universidad de Zaragoza, Aragon Institute of Engineering Research, Zaragoza, Spain
}

\begin{abstract}
The goal of this paper is to describe the effects of simple electro-mechanical coupling in isotropic two-dimensional (2D) cardiac tissue. To this aim, we show that the NashPanfilov two variable model [PRL 95, 258104 (2005)] for electrical activation, which couples active stress directly to transmembrane potential, can be reduced in the linearly elastic regime to an excitatory system with global coupling. In the linear limit, numerical simulations of both models give the same dynamic evolution, including the appearance of an ectopic focus with origin at the center. Indeed, after an initial excitation, mechano-electrical coupling can generate sustained oscillations in the form of successive waves originated at the center. These oscillations have a large basin of attraction for different sample lengths and values of the stretching current, specially when the recovery time of the excitatory cells is short. We finally present and discuss the appearance of oscillatory waves whose origin is not the center of the $2 D$ sample but a ring of tissue around it. These waves appear spontaneously under some conditions even when the first excitation is generated at the center.
\end{abstract}

\section{Introduction}

One of the best investigated aspects of cardiac dynamics is the electrical activity in the heart. The sinoatrial node, a collection of cells situated on the right atrium, next to the vena cava, generates electrical impulses with a period mainly regulated by the sympathetic and the parasympathetic nervous system. This initial activity is propagated in the atrium, passes through the atrioventricular node, proceeds through the bundle of His to the Purkinje fibers, reaching finally the whole ventricle [1]. The dynamics of the currents involved at the cellular level is well established and its macroscopic propagation can be accounted for obtaining experimentally the electric diffusion tensor at the different points of the heart [2].
Furthermore, the mechanism by which electric propagation affects the elastic properties of the cell is well known. After the cell is depolarized, there is an influx of calcium ions inside the cell. This turns on a set of complex mechanisms which unblock the connection between the actin and myosin proteins present in the sarcomere of the cell. The bonding of those proteins generates internal forces which change the viscoelastic properties of the cell in the socalled active state [1]. Unfortunately, this knowledge of the cellular mechanisms is very difficult to translate to a macroscopic level [3]. There are different reasons for this difficulty. First, the passive properties of the heart as a viscoelastic material when no electrical activity is present are not generally known, due to the high anisotropy of the tissue, and the difficulty in performing experiments to measure tri-axial viscoelastic constants in tissue. Second, it is even more difficult to asses the viscoelastic properties in the active state, and to relate them with cell properties, given the combination of passive elements (collagen proteins, fibroblasts) and active elements (cardiomyocytes), which change in a highly history-dependent way.

Furthermore, recent studies have shown the existence of a mechano-electric feedback where the stretching of the heart affects the propagation of the electric signal, due to specific ion channels which are stretch-dependent (SAC) $[4,5]$. This is, the ability of ions to pass through these channels depends on the level of stretching of the cell membrane. Different simplified models have been proposed that, taking into account the viscoelastic properties of cardiac tissue and the strength of the mechano-electric feedback, analyze the kind of dynamics and new behavior that may ensue [6,7]. A good example is the Nash-Panfilov (NP) model [6], which describes electro-mechanical coupling in isotropic cardiac tissue at the most basic level.

In this contribution we show that, as long as deformations are small, mechano-electric feedback in the 2D radially symmetric Nash-Panfilov model introduces a global feedback term in the equation for the transmembrane potential. We will justify the generality of this model as the 
most simple way to incorporate electro-mechanic dynamics in the heart. Later we demonstrate that the model reproduces the oscillatory regime of the Nash-Panfilov model where consecutive pulses are generated at the center [8]. We also uncover the appearance of spontaneous oscillations whose focus are not the center of the tissue but an annular ring. We finally conclude discussing the limitations and possible future lines of research.

\section{Methods}

Our starting point is the Nash-Panfilov model for the transmembrane potential propagation with electromechanic feedback [6]:

$$
\begin{aligned}
& \partial_{t} V=D \nabla^{2} V-\kappa_{h} V(V-1)(V-a)-h V-I(j) \\
& \partial_{t} h=\epsilon(V)\left(\kappa_{h} V-h\right) \\
& \partial_{t} T_{a}=\epsilon(V)\left(\kappa_{T} V-T_{a}\right) \\
& \partial_{X_{M}}\left(T^{M N} \frac{\partial x_{j}}{\partial X_{N}}\right)=0 .
\end{aligned}
$$

Here $V$ is the normalized dimensionless transmembrane potential, $h$ a recovery gate, $X_{i}$ the fixed reference coordinates, $x_{i}$ the material coordinates, and $D$ the diffusion constant. The Laplacian operator reads $\nabla^{2} V=\partial_{X_{M}}\left(\sqrt{C} C_{M N}^{-1} \partial_{X_{N}} V\right)$, with $C_{M N}=$ $\left(\partial x_{k} / \partial X_{M}\right)\left(\partial x_{k} / \partial X_{N}\right)$ the right Cauchy-Green deformation tensor. Mechanical feedback is provided by the stretch-activated current

$$
I_{g}=g(V-1)(\sqrt{C}-1) \Theta(C-1),
$$

which only acts when the cell locally stretches, with $C=$ $\operatorname{det}\left(C_{M N}\right)$, and $\Theta(x)$ the Heaviside function.

To obtain the deformation $C$, a set of equations is needed relating the contraction of the medium with the change of transmembrane potential. Eq. (3) expresses the dependence of the internal tension generated in the cell, $T_{a}$, with the transmembrane potential $V$. The active tension in the system increases with $V$, with a delay fixed by $\epsilon(V)$, given by $\epsilon(V)=\epsilon_{a}$, for $V>a$ and $\epsilon(V)=\epsilon_{b}$, for $V<a$. Then, an isotropic hypoelastic model is formulated to describe the mechanical properties of tissue. The total stress is the sum of an active and a passive component

$$
T^{M N}=\frac{1}{2}\left(\frac{\partial W}{\partial C_{M N}}+\frac{\partial W}{\partial C_{N M}}\right)+T_{a} C_{M N}^{-1} .
$$

The strain energy function is given by

$$
W=c_{1}\left(I_{1}-3\right)+c_{2}\left(I_{2}-3\right),
$$

with $I_{1}=\operatorname{tr}(C)$ and $I_{2}=\operatorname{det}(C)$ the first and second invariants of $C_{M N}$. This model specifies the mechanical properties of the fiber relating the active stress $T_{a}$ in the medium, its passive properties, and the displacements generated in the fiber given by $C_{M N}$.

When active tension is small compared with passive stresses, the elastic equations of the model can be reduced to the standard 2D equations of linear elasticity. Let $x_{k}=X_{k}+u_{k}$, so $\partial x_{k} / \partial X_{M}=\delta_{k M}+\partial u_{k} / \partial X_{M}$. We will assume that $\partial u_{k} / \partial X_{M} \ll 1$ and $T_{a} \ll c_{1}, c_{2}$, so active tension is small compared to the elastic moduli. Under these assumptions, Eqs. (4), (6) and (7) become

$$
c_{1} \nabla^{2} \vec{u}+c_{2} \vec{\nabla}(\vec{\nabla} \cdot \vec{u})+\vec{\nabla} T_{a}=0 .
$$

In the following section we show simulations of the model defined by Eqs. (1)-(3) and (8), thus using this linear equation as the material constitutive relation. We solve the coupled electro-mechanical problem in a rectangular $2 \mathrm{D}$ tissue where the basic electric model for the cell and the constitutive equations are implemented as user subroutines in the multipurpose finite element software ABAQUS. A semiimplicit formulation is used. The gate variable $h$ and the internal tension $T_{a}$ are integrated explicitly, while an implicit formulation is used to solve the coupled problem defined by Eq. (1) and (8). Temporal and spatial discretization are set to $\mathrm{dt}=1 \mathrm{msec}$ and $\mathrm{dx}=0.8 \mathrm{~mm}$ respectively.

For a radially symmetric field the system can be further simplified. In that case, Eq. (8) reduces to

$$
\frac{\partial}{\partial r}\left[\frac{1}{r} \frac{\partial}{\partial r}\left(r u_{r}\right)\right]+\frac{\partial T_{a}}{\partial r}=0,
$$

where $u_{r}$ is the component of the displacement in the radial direction. Solving the former equation for $u_{r}$, we obtain

$$
u_{r}=\frac{1}{c_{1}+c_{2}}\left[r \frac{A}{2}-\frac{1}{r} \int_{0}^{r} r^{\prime} T_{a}\left(r^{\prime}\right) d r^{\prime}\right]
$$

with an arbitrary constant $A$ that has to be determined imposing the boundary conditions. For fixed boundaries in a circular domain, $u_{r}=0$ at $r=R$, this constant takes the value

$$
A=\frac{2}{R^{2}} \int_{0}^{R} r^{\prime} T_{a}\left(r^{\prime}\right) d r^{\prime}=\bar{T}_{a}
$$

where $\bar{T}_{a}$ denotes the average value of $T_{a}$ over the circular domain. So, finally

$$
u_{r}=\frac{1}{c_{1}+c_{2}}\left[r \frac{\bar{T}_{a}}{2}-\frac{1}{r} \int_{0}^{r} r^{\prime} T_{a}\left(r^{\prime}\right) d r^{\prime}\right] .
$$

This result can be used to simplify Eq. (5) noting that $C=\operatorname{det}\left(C_{M N}\right) \simeq 1+2 \vec{\nabla} \cdot \vec{u}$, and $\sqrt{C} \simeq 1+\vec{\nabla} \cdot \vec{u}$. Using our previous expression, we obtain:

$$
\vec{\nabla} \cdot \vec{u}=\frac{1}{r} \frac{\partial}{\partial r}\left(r u_{r}\right)=\frac{\bar{T}_{a}-T_{a}(r)}{c_{1}+c_{2}}
$$


so Eq. (5) finally becomes

$$
I_{g}=\frac{g}{c_{1}+c_{2}}(V-1)\left[\bar{T}_{a}-T_{a}(r)\right] \Theta\left[\bar{T}_{a}-T_{a}(r)\right] .
$$

In other words, in the linear elastic regime under radially symmetric conditions, the Nash-Panfilov model can be reduced to an excitatory system - similar to the FitzHughNagumo model- with global coupling. This same procedure could be followed for a more complex model where the transmembrane potential $V$, and the active tension $T_{a}$, are controlled by multiple gates $\vec{h}=\left(h_{1}, h_{2}, \ldots\right)$, related to different ionic currents. In this case, the SAC current would have a general dependence of the form $I_{g}\left(V, \vec{h}, T_{a}, \bar{T}_{a}\right)$.

For the rest of the paper, we will consider the same $\epsilon(V)$ for both $h$ and $T_{a}$, so that $T_{a}=\left(\kappa_{T} / \kappa_{h}\right) h$ can be introduced in $I_{g}$ [defined by Eq (14)], and the dynamics is effectively defined by only two variables $(V, h)$, evolving according to Eqs. (1) and (2). We have simulated this simplified two-variable model in a circular tissue using a finite difference scheme with $0.1<\mathrm{dx}<0.8 \mathrm{~mm}$ and $\mathrm{dt}=0.1$ msec. We reproduce the same results as solving the full linear Eq. (8) with the ABAQUS solver, if we take the diameter of the circle to be the same as the side of the square, and provided that the radial symmetry is conserved. This indicates that, in this situation, boundary effects are not very important (we only considered systems larger than 3.5 $\mathrm{cm})$.

\section{Results}

The linear elastic and radially symmetric case presents, under a broad range of parameters, an oscillatory regime where successive pulses are generated from the center of the tissue. The evolution of $V$ and $h$ for the center point in the cell is oscillatory, as it is the evolution of global variables, i.e. the average values of transmembrane potential $V$ and gate $h(\bar{V}, \bar{h})$. In Fig 1 we show a characteristic pattern obtained at $t=50,150,230,270 \mathrm{~ms}$ after initial excitation, showing the presence of a pulse advancing radially outwards towards the boundary of the tissue, while another pulse appears at the center. This kind of pattern is the same as those obtained in [8] solving the model with the full elastic equations (1)-(7).

To explain this behavior we should understand the mechanism behind the oscillatory regime, and specifically, the appearance of successive waves. This question can be addressed studying the nullclines of Eqs. (1) and (2) at the source point, as has been done in the case of a onedimensional fiber [9]. Once the wave has originated (Fig. 1a), the variable $h$ increases above the average value in tissue $\bar{h}$. At this point, the stretch activated current closes at the origin [from Eq. (14); recall that $T_{a}=\left(\kappa_{T} / \kappa_{h}\right) h$ ], and the system begins to recover. In this situation the only
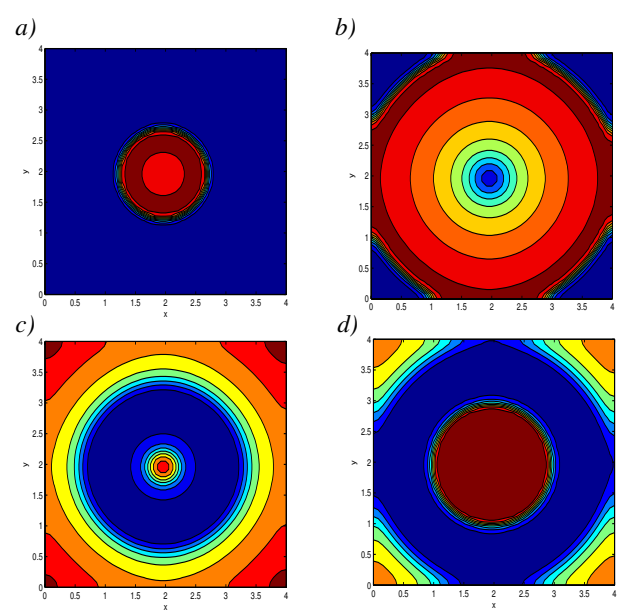

d)

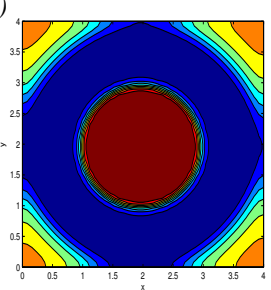

Figure 1. Transmembrane potential in a rectangular patch of tissue of $4 \mathrm{~cm}$ per side after a) 50, b) 150 , c) 230 and d) $270 \mathrm{~ms}$ of initial excitation at the center of the tissue, obtained using the ABAQUS code with parameters $D=0.5$ $\mathrm{mm}^{2} / \mathrm{s}, \kappa_{h}=1000 \mathrm{~s}^{-1}, \kappa_{T}=1 \mathrm{MPa}, a=0.05, \epsilon_{a}=3$ $\mathrm{s}^{-1}, \epsilon_{b}=90 \mathrm{~s}^{-1}, c_{1}=3.85 \mathrm{MPa}, c_{2}=9.6 \mathrm{MPa}$, and $g=500 \mathrm{~s}^{-1}$. The color code is such that red and blue correspond, respectively, to depolarized and repolarized tissue. The appearance of a new wave growing radially from the center while the first one reaches the end of the system can be clearly observed. The same basic results are obtained if we use the approximation given by Eq. (14) in a circular patch of tissue of diameter $4 \mathrm{~cm}$ (see Methods section).

stable point is the trivial steady state $V=0, h=0$. If the relaxation is such that $\bar{h}$ remains significatively above zero because some other part of the tissue is active (i.e. a wave is still propagating), the trivial state suddenly becomes unstable due to the opening of the stretch activated current $I_{g}$, pushing again the voltage towards depolarization. This generates another wave which propagates along tissue closing the oscillation loop.

In order to obtain this oscillatory behavior, the value of $g$ has to be large enough so that the nullclines intersect in the unstable branch of the cubic nullcline, generating an unstable fixed point. Furthermore, a global oscillatory system requires $\bar{h}$ to oscillate around a fix high value, otherwise the stretching current would not be activated at the excitation point. The appearance of new waves at one point has to be compensated with the disappearance of waves which reach the boundary. At the same time, the system has to be large enough or $\epsilon_{b}$ high enough, so that $h$ at the origin falls sufficiently below $\bar{h}$ to have a strong stretch current which can sustain the oscillation.

The appearance of successive waves with origin at the center is not the only non-trivial attractor we find. Keeping the exact same parameters as before, but doubling the 


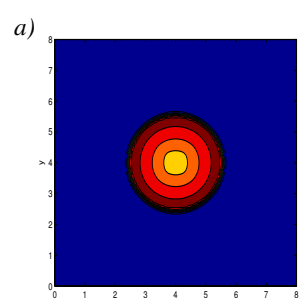

c)
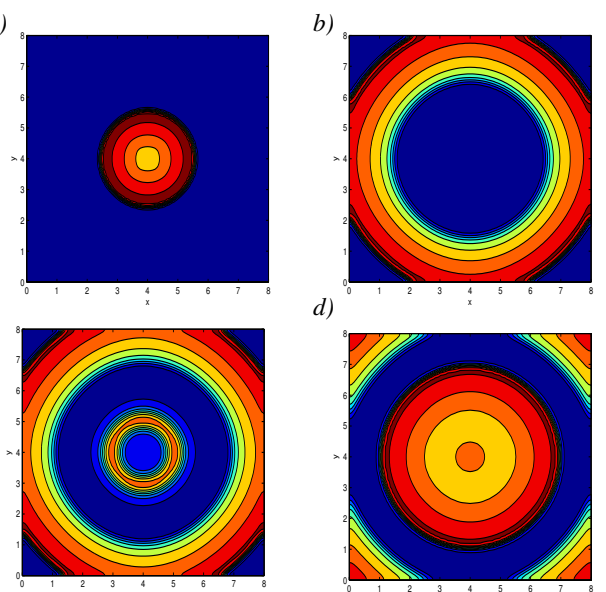

d)

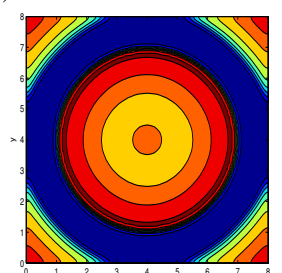

Figure 2. Four pictures of the evolution of electrical activity in a rectangular patch of tissue, showing the presence of an attractor where the successive fronts are generated in an annular ring, and not at the center of the cell. Pictures a) to d) are taken at times $t=100,300,330$, and $400 \mathrm{~ms}$, after which the pattern is repeated with a periodicity of $\sim 200$ ms. Same parameters as in Fig. 1 but with a system size of $8 \times 8 \mathrm{~cm}$.

size of the system, we obtain a succession of patterns as those presented in Fig. 2. Starting with an excitation at the center (Fig. 2a), we clearly see that the starting point of the next wave is not the same, but an annular ring around the center (Fig. 2c). From there, an inward and an outward front advance until they reach the center and the boundary of the system (Fig. 2d). This process is repeated continuously and successive annular excitations appear. This pattern indicates that there is characteristic length scale behind the front, arising from an interplay of diffusion, size of the system, and the recovery processes of the slow variable $h$.

\section{Discussion and conclusions}

Concluding, we have been able to understand the underlying physical mechanism of the oscillatory regime in the Nash-Panfilov model in 2D, demonstrating its equivalence to a FitzHugh-Nagumo type model with global coupling, and studying its nullclines in the radial symmetric case. We have shown that boundary effects which slightly break the radial symmetry do not affect the characteristic patterns, as long as the initial conditions and subsequent evolution keep this radial symmetry.

Our simplification provides a basic framework to understand mechano-electric coupling in the heart. In the more general case without radial symmetry we can still make the linear approximation and solve the elastic equations. The exact mathematical problem will be cumbersome. Generally, the deformation tensor $C_{M N}$ can be written in terms of non-local integrals, being the kernel the Green function of the linear elastic problem with the appropriate boundary conditions. The more general case, thus, implies global coupling but not as simple averages, but as convolutions of $T_{a}$.

One of the main limitations of our formulation, in order to understand the basic ingredients of mechano-electric coupling in $2 \mathrm{D}$ experiments, is that it does not incorporate anisotropy of the tissue. This would affect the formulation of stress (both active and passive), the stretch current, and the diffusion tensor. Our future aim is to develop a similar simplified description which takes into account these effects. We hope that it will be equally valuable to understand anomalous heart behavior.

\section{Acknowledgements}

We thank F.H. Fenton and E. Cherry for valuable discussions. This study was supported by MEC (Spain), under project FIS2005-06912C02-01, under network DPI2007-30607-E, and under the Ramón y Cajal (B.E. and J.F.R.) and Juan de la Cierva (E.A-L.) programs.

\section{References}

[1] Frank B. Sachse. Computational Cardiology: Modeling of Anatomy, Electrophysiology, and Mechanics, Springer-Verlag Berlin and Heidelberg, 2004.

[2] Roth BJ. Electrical conductivity values used with the bidomain model ofcardiac tissue. IEEE Transactions on Biomedical Engineering 1997;44:326-328.

[3] Nash MP, Hunter PJ. Computational Mechanics of the Heart. Journal of Elasticity 2000;61:113141.

[4] Kohl P, Ravens U Eds., Special issue on Mechano-Electric Feedback and Cardiac Arrhythmias. Progress in Biophysics and Molecular Biolology 2003;82: Nos.1-3.

[5] Kohl P, Hunter P, Noble D. Stretch-induced changes in heart rate and rhythm: clinical observations, experiments and mathematical models. Progress in Biophysics and Molecular Biolology 1999;71:91-138.

[6] Nash MP, Panfilov AV. Electromechanical model of excitable tissue to study reentrant cardiac arrhythmias. Progress in Biophysics and Molecular Biolology 2004;85:501-522.

[7] Bini D, Cherubini C, Filippi S. Viscoelastic Fitzhugh-Nagumo models. Physical Review E 2005;72:041929.

[8] Panfilov AV, Keldermann RH, and Nash MP. Self-Organized Pacemakers in a Coupled Reaction-Diffusion-Mechanics System. Physical Review Letters. 2005;95:258104.

[9] Alvarez-Lacalle E, and Echebarria B. unpublished.

Address for correspondence:

Dr. Enrique Alvarez Lacalle

Av Doctor Marañon 44-50,

EPSEB Departament Fisica Aplicada, UPC, Barcelona, Spain

e-mail: enrical@fa.upc.edu 